\title{
The time course of response inhibition in masked priming
}

\author{
ANGELIKA LINGNAU and DIRK VORBERG \\ Technical University Braunschweig, Braunschweig, Germany
}

\begin{abstract}
In two experiments, we studied the temporal dynamics of the response time effects of masked visual prime stimuli, as a function of stimulus eccentricity and size. Experiment 1 factorially varied prime-target congruency, eccentricity, and mask-target stimulus onset asynchrony. Early facilitative and late inhibitory effects of congruency were observed at all eccentricities, with temporal dynamics modulated by eccentricity. To test whether this dependence on eccentricity is due to cortical magnification, Experiment 2 varied stimulus size as well. Response inhibition time courses were influenced by size and eccentricity jointly, with no discernible difference when stimuli were matched for cortical magnification. Analysis of the individual time course data revealed that the timescale of inhibition changes with the strength of the cortical representation of the prime stimulus. This imposes constraints on possible models.
\end{abstract}

It is well known that invisible prime stimuli may affect responses to subsequent target stimuli (Klotz \& Wolff, 1995; Neumann \& Klotz, 1994; Vorberg, Mattler, Heinecke, Schmidt, \& Schwarzbach, 2003). In the masked priming paradigm, participants react faster and commit fewer errors when masked prime and target are congruent with respect to a common feature that specifies the response (e.g., the direction of an arrow), as compared with trials in which prime and target are incongruent (e.g., prime and target point in opposite directions). The positive response time (RT) difference between incongruent and congruent trials is termed the (net) congruency or priming effect.

\section{Response Inhibition}

Surprisingly, a minor procedural change in the experimental procedure leads to opposite results: Inserting a delay between mask and target may reverse the polarity of the priming effect, so that congruent primes lead to slower and more error-prone responses than do incongruent primes. Schlaghecken and Eimer (1997), who first discovered and described this reversal of the congruency effect, called it the negative compatibility effect, in contrast with the normal priming effect (which they refer to as the positive compatibility effect). ${ }^{1}$

Eimer and Schlaghecken (1998) explained their findings as evidence for active suppression of the partial response activation that is elicited by a masked prime and supported their account with lateralized readiness potential (LRP) data. They observed that on congruent trials,

We are grateful to P. Zwitserlood and K. Schütz for discussion and many helpful suggestions. A.L. was supported by the Studienstiftung des Deutschen Volkes (PhD grant). Correspondence concerning this article may be sent to D. Vorberg, Department of Psychology, Technical University Braunschweig, Spielmannstr. 19, 38106 Braunschweig, Germany (e-mail: d.vorberg@tu-bs.de). about $200 \mathrm{msec}$ from prime onset the LRP shows a partial activation of the response congruent with the prime, but this activation is then replaced by an inhibitory phase that leads to activation of the alternative response. Replicating Schlaghecken and Eimer's (1997) original findings, Klapp and Hinkley (2002) also showed that the inhibitory response is strongly tied to the presence of the mask, with positive effects of congruency only when the mask is omitted.

In this article, we are concerned with the inhibitory mechanism that seems to underlie the reversal from positive to negative effects of prime-mask congruency and how this inhibition evolves over time. In particular, we focus on whether inhibition is different for stimuli presented centrally or peripherally (see below). We feel that it is important to distinguish the underlying inhibitory mechanism from the empirical phenomenon; thus, we use the term negative congruency effect (NCE) for the observed polarity reversal of the congruency effect only, but refer to the more general phenomenon and its underlying mechanism with the term response inhibition. The reason for this distinction is that response inhibition does not necessarily imply negative congruency effects. Obviously, a polarity reversal of the congruency effect is sufficient but not necessary for the existence of response inhibition: Negative effects only arise if the RT costs due to response inhibition exceed the RT benefits due to prime congruency. Thus, an observed negative congruency effect implies response inhibition, but the converse is not true. As we shall show here, there may be evidence other than negative congruency effects that suggest the occurrence of response inhibition.

\section{The Central-Peripheral Asymmetry}

Surprisingly, Schlaghecken and Eimer (1997, 2000) found negative effects for masked primes presented centrally, but only positive effects for peripheral primes. To 
account for this, they postulated a fundamental difference between central and peripheral visual processing, with inhibition for central stimuli only. Subsequently (Schlaghecken \& Eimer, 2002), negative congruency effects were observed for peripheral primes also if the delay between prime and mask was sufficiently long. This led the authors to argue that whether inhibition is evoked or not depends on the strength of the sensory representation of the prime, with weaker representations in the periphery than in the central visual field. Assuming that "only strong partial response activations ... are subject to active inhibition, whereas weaker activations remain below a hypothetical inhibition threshold," the centralperipheral asymmetry was explained as reflecting the fact that "motor activations triggered by foveal primes are more likely to reach the inhibition threshold than are motor activations triggered by peripheral primes" (Schlaghecken \& Eimer, 2002). They concluded that their experiments, which investigated how the negative congruency effect depends on the perceptual strength of peripheral primes, "provide strong support for the inhibition threshold account" (p. 160). This conclusion was based on the failure to find negative congruency effects for central primes when they were perceptually degraded.

We see the following problems with these arguments: (1) As pointed out above, a failure to observe a polarity reversal of the congruency effect need not imply the absence of inhibition. (2) Even if negative effects exist, they will not be detected if searched for in the wrong place. That is, Schlaghecken and Eimer (2002) may have overlooked negative congruency effects that occurred at mask-target delays not covered in their experiments. The results reported in this article support our suspicions.

\section{Relevance of Time Course Information}

Eimer and Schlaghecken's (1998) LRP findings show that the temporal dynamics of response inhibition are crucial for its effect on RT. Whether and how strongly inhibition arises therefore depends on the temporal aspects of the stimuli. This suggests that we study the temporal dynamics of priming and inhibition effects parametrically, by systematically varying the durations of and the intervals between prime, mask, and target stimuli.

The advantages of such an approach are illustrated by the findings of Vorberg et al. (2003), who studied the temporal dynamics of masked priming in a metacontrast masking paradigm in which the same stimulus served both as mask and as target. Contrasting the time course of priming with that of perceptual masking, they demonstrated that response priming is dissociated from conscious awareness of the prime stimuli: Net priming was found to increase with stimulus onset asynchrony (SOA) between prime and mask, with identical slopes under conditions when prime recognition performance was either at chance level or was better than chance but followed a U-shaped time course within the SOA range tested.

Under similar conditions but with separate mask and target stimuli, Vorberg (1998) studied the time course of response inhibition. Whereas prime-mask SOA played a role at small mask-target SOAs only, varying mask-target SOA revealed dramatic inhibitory effects. The initially positive RT difference turned negative for mask-target SOAs between 100 and $150 \mathrm{msec}$, with a second sign reversal toward positive congruency effects at still larger SOAs. Remarkably, in the SOA range in which responses were still faster on congruent than on incongruent trials, mean congruent RT increased with mask-target SOA, whereas mean incongruent RT actually decreased with SOA in the same window. This picture mirrors Eimer and Schlaghecken's (1998) LRP findings obtained with pattern masking and suggests that the particular type of backward masking (pattern vs. metacontrast) is not essential for response inhibition to arise.

Given this dependence of the response inhibition effect on the temporal stimulus conditions, it is obvious that experiments with mask-target SOA fixed at some arbitrarily chosen value risk pitfalls. This idea crucially applies when response inhibition effects of peripheral and central primes are compared. (1) Can we guarantee that no response inhibition would have shown up at some other SOA? (2) What is the single appropriate SOA value, when the time course of response inhibition is subject to substantial variability between participants, both quantitatively and qualitatively (Vorberg, 1998)?

\section{Aims and Overview of the Present Study}

Time course information may help overcome the problem that response inhibition is not necessarily accompanied by a polarity reversal of the congruency effect. In the experiments reported here, we assessed the temporal dynamics of response inhibition for stimuli at increasing distances from the visual center, thus testing the hypothesis that peripheral stimuli do elicit inhibition, but with different temporal dynamics. We show that problems of interindividual variability in temporal dynamics are reduced if one follows the well-established psychophysical tradition of presenting and analyzing individual performance in addition to average results.

With Schlaghecken and Eimer (2002), we assume that the strength of the sensory representation of stimuli differs with eccentricity, with weaker representations for primes in the periphery as compared with the central visual field. The strength of the representation should affect the dynamics of response inhibition. We expected to find evidence of response inhibition for peripheral stimuli also, but with a different time course from that for central stimuli.

Why and how should the temporal dynamics of inhibition change with eccentricity? A plausible hypothesis is based on cortical magnification. Representations of peripheral stimuli are almost certainly weaker because the amount of neuronal space dedicated to central stimuli increases as one moves up the visual pathway from the retina to the lateral geniculate nucleus and the visual cortex (DeValois \& DeValois, 1988; Sereno et al., 1995). As a consequence, peripheral visual stimuli lead to smaller cortical representations and are thus likely to generate less neuronal activity than stimuli presented centrally. 
Because it has less neuronal machinery available, peripheral stimulus processing might take longer and follow time courses that are either shifted (i.e., with onset delayed) or stretched, with maximum positive and response inhibition effects shifted toward larger mask-target SOAs. At small SOAs, this would imply an NCE for central but not necessarily for peripheral primes.

Experiment 1 was designed to test these predictions under conditions that allowed assessing time courses for individual participants. The purpose of Experiment 2 was to test predictions that follow from the cortical magnification account. If the strength of the sensory representation of a stimulus, and thus its time course of inhibition, changes with visual eccentricity due to differential cortical magnification, eccentricity effects must be cancelable by adjusting stimulus size appropriately. This idea leads to two related predictions: (1) Varying stimulus size while keeping eccentricity fixed should produce response inhibition effects analogous to those from varying eccentricity of constant-sized stimuli. (2) Stimuli equated for cortical magnification should produce identical response inhibition time courses.

\section{EXPERIMENT 1}

In this experiment, we studied the time course of response priming and inhibition for stimuli presented at vertical eccentricities at or beyond the $2.2^{\circ}$ boundary that limits the range of response inhibition effects according to Schlaghecken and Eimer (2000). We pre- dicted that in the visual periphery, positive priming and response inhibition build up more slowly and occur at larger SOAs. As in our earlier studies (Vorberg, 1998, 2000; Vorberg et al., 2003; Vorberg, Mattler, Heinecke, Schmidt, \& Schwarzbach, 2004), we masked the prime stimuli by metacontrast rather than by pattern masking.

\section{Method}

Participants. Six female psychology students between 19 and 28 years of age participated in the experiment, either as a course requirement or for a payment of $€ 5$ per session. All participants had normal or corrected-to-normal vision; 5 of them were right-handed, and 1 was left-handed.

Stimuli. Each trial began with the presentation of a fixation cross at the center of the screen, which remained visible until the target stimulus disappeared. On each trial, a sequence consisting of a prime stimulus (14-msec duration), a mask stimulus (112-msec duration), and a target stimulus (112-msec duration) was presented either above or below fixation, with position randomly selected. To prevent eye movements before the target stimulus appeared, each prime was accompanied by a neutral prime with rectangular shape at the complementary position, which was also followed by its mask (see Figure 1).

The prime was a small left- or right-pointing arrow, fitting into the inner cutout of the mask (see Figures 2A and 2B). Neutral prime stimuli were constructed by superimposing left- and right-oriented primes. The outer shape of the mask was rectangular. The target was a large left- or right-pointing arrow, with a cutout large enough for the mask to fit in without touching the inner contours of the target. On congruent trials, prime and target pointed in the same direction, on incongruent trials in opposite directions.

Stimuli were presented in black on a white background (see Figure 2 for details). They appeared at eccentricities of $1.48^{\circ}$ ("near"),

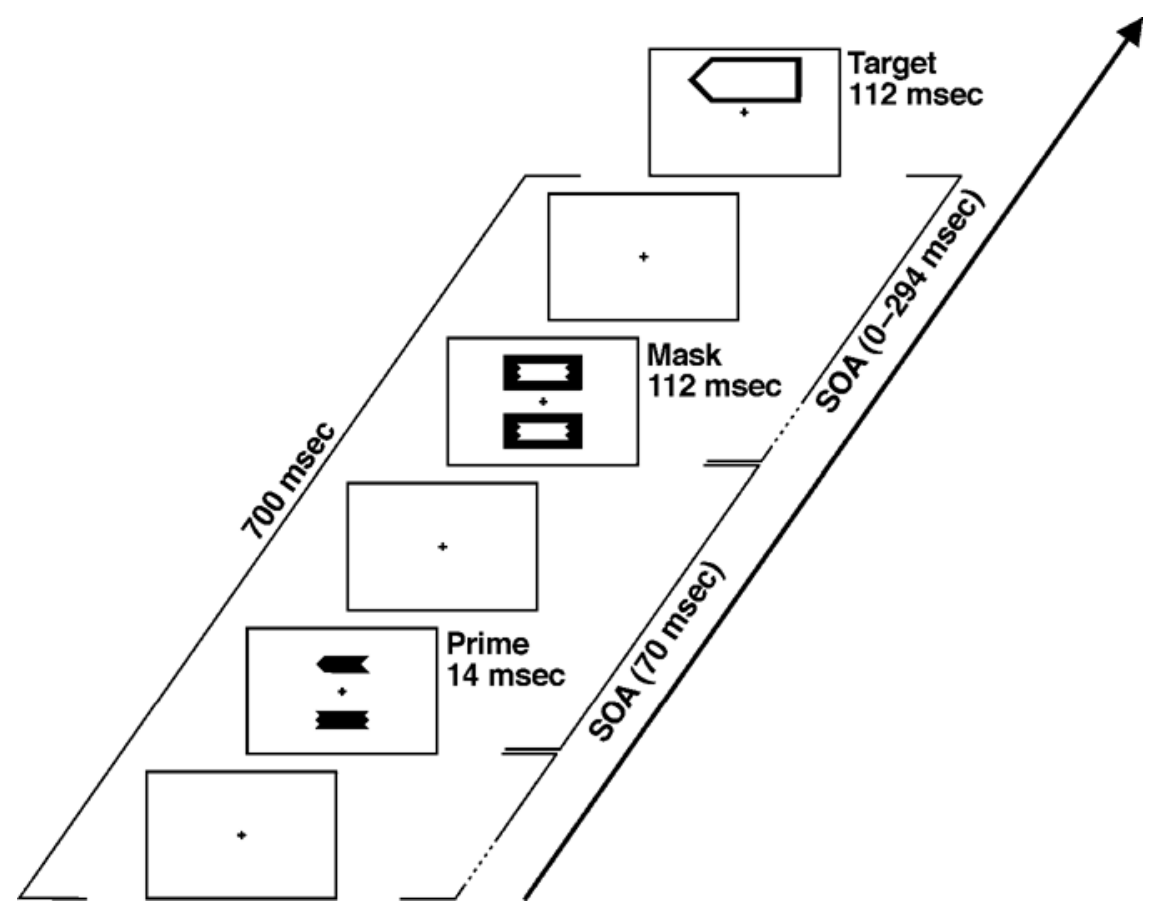

Figure 1. Temporal sequence of prime, mask, and target stimuli on a typical experimental trial. Congruent or incongruent primes were always accompanied by a neutral prime at the complementary location. 


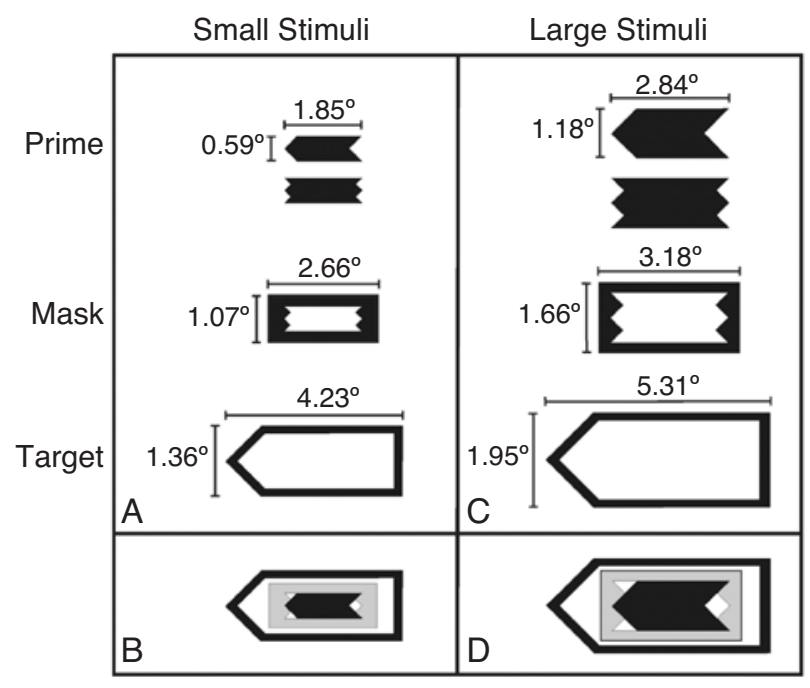

Figure 2. (A, C) Stimuli used as primes, masks, and targets. (B, D) Spatial relations between stimuli, demonstrated by superimposing them, with primes fitting exactly into mask cutouts. Small stimuli were used only in Experiment 1.

$2.96^{\circ}$ ("medium"), or $4.43^{\circ}$ ("far") from the center of the screen. Prime-mask SOA was constant at $70 \mathrm{msec}$, and mask-target SOA varied between 0 and $294 \mathrm{msec}$ in steps of $42 \mathrm{msec}$. Trial events were timed so that the target stimulus always appeared $700 \mathrm{msec}$ after the fixation cross.

The extent to which primes remained outside awareness was not of theoretical concern here. Therefore, we did not assess prime visibility in psychophysical detection or recognition tasks. However, under spatial and temporal conditions similar to the "near" condition, Vorberg et al. (2003) found that participants could not report the identity of the primes at prime-mask SOAs from 14 to $70 \mathrm{msec}$, even after extended practice of more than 3,000 trials. This finding agrees with the fact that none of the participants of the present experiments mentioned having noticed the masked prime arrows.

Design. Prime-target congruency (congruent or incongruent), stimulus eccentricity (near, medium, or far), and mask-target SOA $(0,42,84,126,168,210,252$, or $294 \mathrm{msec})$ were combined factorially, varying randomly from trial to trial. As participants typically prepare for an average SOA, such a broad SOA range could be problematic. Therefore, SOA was blocked in small SOA $(0,42,84$, or $126 \mathrm{msec})$ and large SOA $(168,210,252$, or $294 \mathrm{msec})$ blocks, which alternated regularly.

Procedure. The task was to respond to the direction of the target arrow by pressing the corresponding response button with the left or right index finger. The participants were instructed to respond as quickly as possible while keeping their overall error rate below 5\%. Visual feedback was given on error trials. The participants were not informed of the existence of the masked primes.

Experimental blocks consisted of 48 trials preceded by 3 warmup trials (excluded from data analysis). The participants started trial blocks at their discretion. Information about mean RT and error percentage was given after each block. Prior to the main experiment, the participants performed two practice sets of 20 trials each.

The participants performed seven sessions each, distributed over 2 successive weeks. A session consisted of 16 blocks, with a 5-min break after the eighth block. There were 112 replications per condition and participant.

Data analysis. RTs on correct trials were summarized by trimmed means per participant and condition, trimming $10 \%$ each from above and below (Wilcox, 1997). Repeated measures $2 \times 3 \times 8$ analyses of variance (ANOVAs) were computed separately for the trimmed RT means and the arcsine-transformed error rates, with congruency, eccentricity, and SOA as orthogonal factors. Degrees of freedom were adjusted by the Huynh-Feldt procedure when Mauchly's tests indicated violation of the sphericity assumption (associated $p$ values denoted as $\left.p_{\mathrm{HF}}\right)$.

\section{Results}

Figure 3 shows mean RTs and error rates on congruent and incongruent trials, as functions of SOA and eccentricity. As can be clearly seen, positive congruency as well as response inhibition effects were found at all three eccentricities.

On congruent trials, RT and error rate varied nonmonotonically with SOA. Responding was fastest at small SOAs (0-42 msec), slowed down at medium SOAs (84-168 msec), and leveled off at large SOAs (210 $294 \mathrm{msec}$ ). This RT increase was accompanied by an error rate increase. The reverse picture was found on incongruent trials, with fastest responses and fewest errors at medium SOAs, but slower responses and more errors at

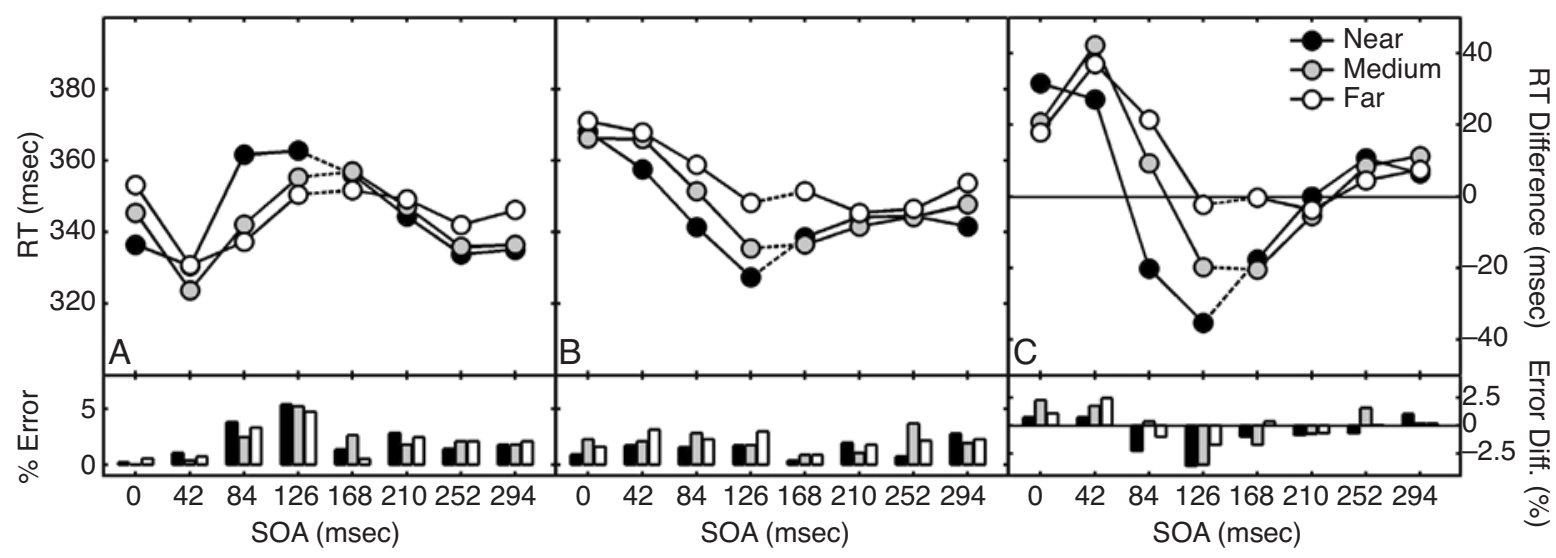

Figure 3. Results from Experiment 1. Mean response times (RTs, top panels) and error rates (bottom panels) for congruent (A) and incongruent (B) trials. Panel $C$ shows the net priming effect $\left(\mathbf{R T}_{\text {incongruent }}-\mathbf{R T}_{\text {congruent }}\right.$ ) and the error rate differences

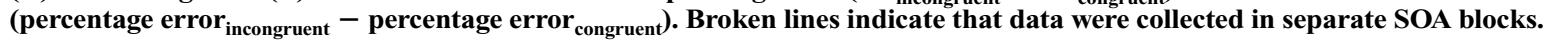


small and large SOAs. The dynamics of priming and inhibition are seen best in the net RT difference $\left(\mathrm{RT}_{\text {incongruent }}-\right.$ $\left.\mathrm{RT}_{\text {congruent }}\right)$ and the corresponding error rate difference (Figure 3C). The typical U-shaped time course was found at all three eccentricities, both for response speed and for accuracy. Most importantly, time courses were modulated by eccentricity. Onset, peak, and trough were more widely separated in time as the stimulus position became more eccentric. This can be seen, for example, by comparing the zero crossings of the RT differences (Figure 3C).

These observations were supported by statistical analyses. Of primary concern is the congruency $\times$ eccentricity $\times$ SOA interaction, which was highly reliable for RT as a dependent variable $[F(14,70)=12.30, p<.0001]$ but not for error rate $[F(14,70)=1.12, p=.355]$. As expected, the interactions of congruency with SOA and eccentricity were reliable as well for both RT [congruency $X$ SOA, $F(7,35)=26.56, p<.0005$; congruency $\times$ eccentricity, $F(2,10)=8.53, p=.007$ ] and error rate [congruency $\times \mathrm{SOA}, F(7,35)=5.35, p<.0001$; congruency $\times$ eccentricity, $F(2,10)=8.53, p=.206]$. The overall effect of eccentricity on RT was small (near, $345 \mathrm{msec}$; medium, $346 \mathrm{msec}$; far, $351 \mathrm{msec}$ ) but statistically reliable $\left[F(2,10)=19.00, p_{\mathrm{HF}}=.001\right]$; the corresponding effect on error rate was not significant $[F(2,10)=$ $\left.3.87, p_{\mathrm{HF}}=.641\right]$.

The crucial question is whether any response inhibition was elicited by primes at the far eccentricity. Obviously, the NCE observed for near primes is mostly due to the fast RT increase with SOA on congruent trials, and less so to its reduction on incongruent trials. Focusing on this paradoxical slowing on congruent trials, we checked with post hoc tests whether far primes did elicit corresponding changes. The results fully confirm the visual impressions: Orthogonal contrasts revealed significant response slowing due to congruent primes at the far eccentricity both at the 126-msec and 168-msec SOAs, in comparison with the average RT at the preceding SOAs $[F(1,107)=6.91, p<$ $.01 ; F(1,107)=5.54, p<.05 ; d f$ s estimated by WelchSatterthwaite approximation-see Howell, 2002, p. 492]. Moreover, RT on these trials changed nonmonotonically with SOA, as revealed by trend analysis (Howell, 2002, p. 408), which yielded highly reliable third- and fourthorder orthogonal contrasts $[F(1,107)=12.40, p<.0001$; $F(1,107)=24.48, p<.0001]$ for far congruent primes, similar to the effects at medium and near distances.

Figure 4 shows the time course data for individual participants - that is, mean RT on congruent trials (left panel) and incongruent trials (middle) and the RT difference (right). For descriptive purposes, cubic splines (computed with MATLAB 6.0) have been fitted to the data. In spite of considerable interindividual differences, there is remarkable qualitative consistency across participants. Importantly, the U-shaped profile typical of the response inhibition effect (Figure 4, right) was observed for each participant at either eccentricity, as was the RT increase on congruent trials within the SOA range from 42 to $126 \mathrm{msec}$, which is in stark contrast to the RT decrease on incongruent trials within this range.
Systematic variability between participants with respect to the onset and offset of the time course under a given experimental condition is bound to distort the temporal dynamics that result when averaging across participants at a given SOA (as in the statistical analyses reported above). To assess whether the onset of response inhibition and its temporal extent change with prime eccentricity, we therefore determined the SOA location of the RT minimum and maximum on each curve; their averages across participants are indicated by the small arrows in Figure 4. There was no significant change in the inhibition onset with eccentricity, nor in the location of the initial RT minimum on congruent trials or of the maximum on incongruent trials. However, the absolute average slope of the time courses between these extremes decreased with eccentricity [congruent trials, 4.47 vs. 3.57 vs. $2.15, \chi_{2}^{2}$ (Friedman rank ANOVA) $=4.33, p=.10$; incongruent trials, 3.35 vs. 2.08 vs. $1.87, \chi_{2}^{2}=7.58, p<$ $.025]$, suggesting that the inhibition time course indeed expands with prime eccentricity. This is also seen in the location of the minimum RT difference, which occurs later with the more peripheral primes (126 msec vs. $147 \mathrm{msec}$ vs. $175 \mathrm{msec}, \chi_{2}^{2}=6.08, p<.05$; see Figure $4 \mathrm{C}$ ).

\section{Discussion}

Experiment 1 showed clear response inhibition effects evolving with mask-target delay. At the smallest masktarget SOA, RT benefits were observed on congruent trials, which turned into RT costs when the target was delayed by 70 to $100 \mathrm{msec}$ from the mask. After even larger SOAs, inhibition gave way to positive congruency effects again. Analogous patterns were seen in the error rates. As predicted, time courses were modulated by eccentricity. Most importantly, however, response inhibition effects did not vanish even for prime stimuli at the $4.4^{\circ}$ eccentricity, which has been claimed to be immune to response inhibition (Schlaghecken \& Eimer, 2000). There was consistent evidence for an inhibitory response at all eccentricities, which can be seen most conspicuously in the paradoxical slowing of responses and increase of error rate on congruent trials at medium SOAs. Even though the sign of the congruency effect did not reverse for the far primes, inhibition could be safely inferred from the fact that on congruent trials, RT and error rate increased with SOA, whereas on incongruent trials RT and error rate decreased within the same SOA range. Obviously, using a fixed-SOA experimental design would have led to the misleading conclusion that response activation was too weak to trigger inhibition at this eccentricity.

Our data suggest that, at least at eccentricities up to $4.4^{\circ}$, an inhibitory response always follows the initially positive effect elicited by a masked prime; however, for more eccentric stimulus locations, the inhibitory response follows a more extended time course with reduced amplitude.

These time course data seem to support the idea that response inhibition effects depend on the strength of the sensory representation of the prime, as suggested by 


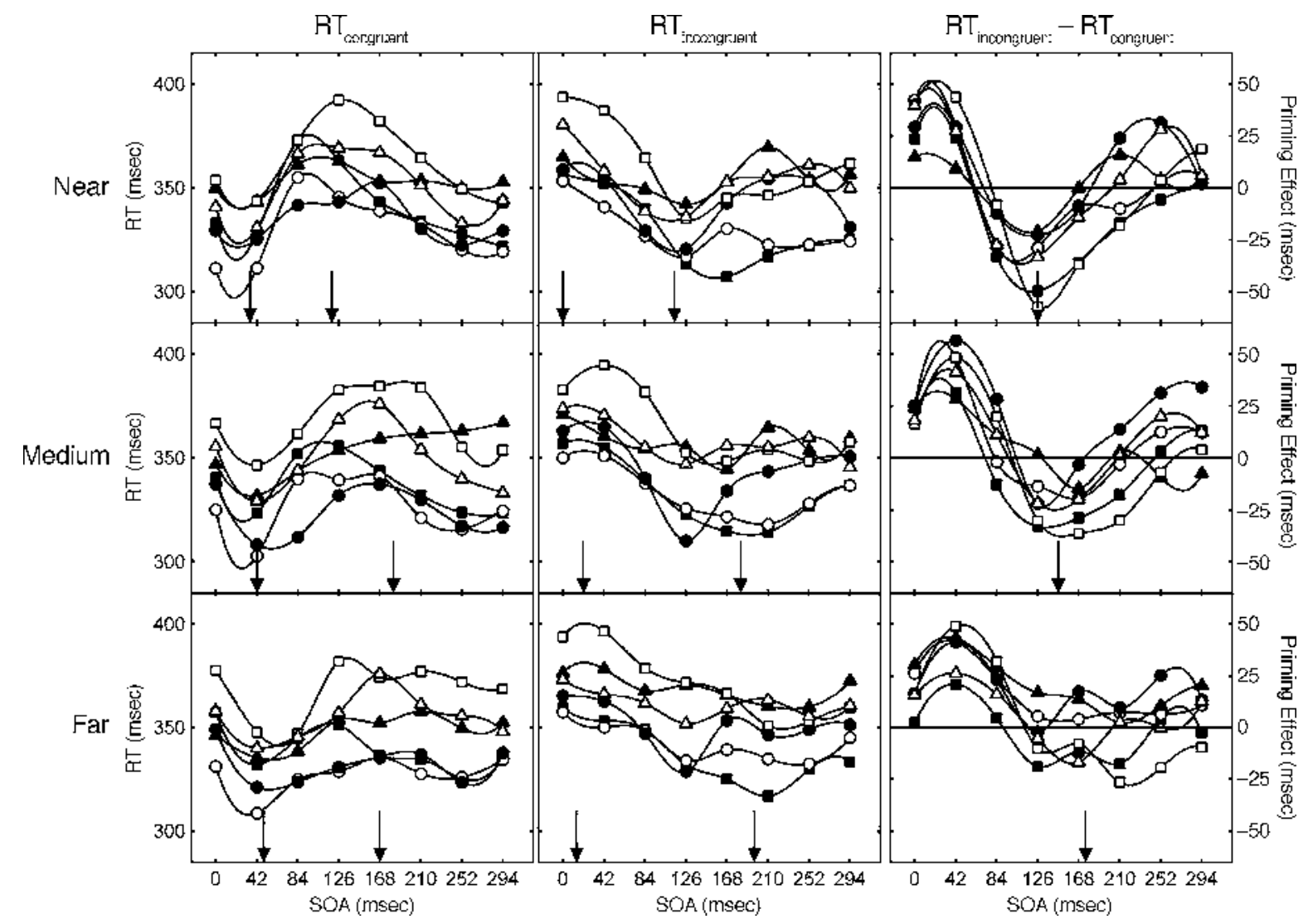

Figure 4. Time courses of individual participants in Experiment 1, with cubic splines fitted to the data. The left and middle columns indicate mean response times (RTs) on congruent and incongruent trials, respectively. The right column shows the net priming effect $\left(\mathrm{RT}_{\text {incongruent }}-\mathrm{RT}_{\text {congruent }}\right)$. Rows indicate prime eccentricity: (top) near $=1.48^{\circ}$; $\left(\right.$ middle) medium $=2.96^{\circ}$; $\left(\right.$ bot- $^{\circ}$ tom) far $=4.43^{\circ}$. Arrows indicate average RT minima and maxima across all participants.

Schlaghecken and Eimer (2002). As a consequence of cortical magnification, peripheral visual stimuli lead to representations that occupy less neural space and generate less neural activity. Accordingly, peripheral stimuli take longer to build up a sufficiently strong representation. If decreases in processing efficiency are accompanied by more variable processing times, as is ubiquitous in neural systems (Dayan \& Abbott, 2001), time courses should be both flattened and stretched for peripheral primes, which is what we observed.

If the cortical magnification account is correct, varying stimulus size while holding eccentricity constant should yield results analogous to those from varying eccentricity for constant-sized stimuli. As a consequence, response inhibition should follow identical time courses for stimuli equated for cortical size. Experiment 2 was designed to test these predictions.

\section{EXPERIMENT 2}

How can we test whether the central-peripheral asymmetry of the response inhibition effect reported by Schlaghecken and Eimer $(1997,2000)$ follows from differential cortical magnification? The straightforward way would be to cancel magnification effects by adjusting the size of the stimuli so that their cortical representations cover same-sized areas in visual cortex, irrespective of eccentricity (Carrasco \& Frieder, 1997). This should eliminate any central-peripheral asymmetry and render the respective time courses identical if the hypothesis is correct.

However, there are technical problems with this approach. Effective metacontrast masking strongly depends on the geometry of the stimuli, which is constrained by the discrete pixel grid of the display and precludes perfect cancellation of cortical magnification differences. Therefore, we used a slightly different approach based on the idea that the crucial variable that determines response inhibition and its time course is neither the size nor the eccentricity of the prime stimulus, but rather the spatial extent of its cortical representation. If this is so, varying eccentricity while keeping prime size fixed should produce effects analogous to those for varying the size of primes presented at a fixed eccentricity. Stated differently, if the central-peripheral asymmetry is a consequence of differential magnification, there should exist an analogous large-small asymmetry in the response inhibition effect, even for primes presented near the fovea. 
We factorially varied prime size and eccentricity and compared the response inhibition effects from small and large primes presented near to or far from the screen center. Sizes were chosen so that the predicted cortical extent was approximately the same for the small-near and the large-far primes. This manipulation should abolish the central-peripheral asymmetry. As before, time courses were assessed by varying mask-target SOA.

\section{Method}

Details were as in Experiment 1, except with the following changes.

Participants. Six new female psychology students, between 20 and 30 years of age, all with normal or corrected-to-normal vision, took part in this experiment. One participant was left-handed, the other 5 were right-handed.

Stimuli and Design. Prime-target congruency (congruent, incongruent), stimulus size (small, large), and eccentricity (near, far) were combined factorially. Small primes, masks, and targets were identical to those of Experiment 1; large stimuli subtended visual angles of $2.84^{\circ} \times 1.18^{\circ}$ (prime), $3.18^{\circ} \times 1.66^{\circ}$ (mask), and $5.31^{\circ} \times$ $1.95^{\circ}$ (target) (see Figures $2 \mathrm{C}$ and 2D). The vertical extents of the small and large primes were in a ratio of 1:2, slightly less than the ratio of 1:2.57 predicted for these eccentricities using inverse magnification (see the Appendix for details). To prevent spatial compatibility effects, horizontal extent was not increased to scale. The two eccentricities were $1.48^{\circ}$ (near) and $4.43^{\circ}$ (far). Mask-target SOA varied between 28 and $196 \mathrm{msec}$ in steps of $56 \mathrm{msec}$. All variables varied randomly within blocks.

Procedure. Experimental blocks consisted of 48 trials preceded by 3 warm-up trials (excluded from data analysis). Each participant performed four sessions with 804 trials each, resulting in 96 replications per condition and participant.

\section{Results}

Figure 5 shows mean RTs (top) and error rates (bottom) on congruent (A) and incongruent (B) trials, as well as the corresponding RT and error rate differences $(\mathrm{C})$, as functions of mask-target SOA, stimulus size, and eccentricity. Overall RT effects of stimulus size (small, $347 \mathrm{msec}$; large, $341 \mathrm{msec}$ ) and eccentricity (near, $342 \mathrm{msec}$; far, $346 \mathrm{msec}$ ) were small but statistically reli- able [size, $F(1,5)=25.59, p<.004$; eccentricity, $F(1,5)=$ $71.19, p<.0001]$. The effect of size was also seen in the error rates [small, $2.6 \%$; large, $3.4 \% ; F(1,5)=13.72$, $p<.014]$, whereas the effect of eccentricity on error rate was not reliable $[F(1,5)=0.68, p=.446]$. There was no overall effect of congruency for RT [congruent, $344 \mathrm{msec}$; incongruent, $343 \mathrm{msec} ; F(1,5)=0.019, p=.895]$ or for error rate [congruent, $3.7 \%$; incongruent, $2.1 \% ; F(1,5)=$ $5.28, p=.07]$.

Of more importance is the RT difference between incongruent and congruent trials, which varied from $30 \mathrm{msec}$ to $-20 \mathrm{msec}$ or $-14 \mathrm{msec}$ as SOA increased from 28 to $196 \mathrm{msec}$. This is the typical evolution of the response inhibition effect with increasing mask-target delay, which was statistically reliable for RT [congruency $\times$ SOA, $F(3,15)=24.72, p<.0001]$ but not for error rate $[F(3,15)=1.86, p=.18]$. As expected, the time course was modulated both by size and eccentricity. The cortical magnification account predicts that size effects on response inhibition are analogous to eccentricity effects, and this was observed. Averaged across eccentricity, there was a large-small asymmetry - that is, large primes resulted in stronger response inhibition effects than did small ones. This asymmetry was even stronger than the corresponding near-far asymmetry obtained by averaging across size. ANOVAs on RT corroborated these observations through the reliable interaction size $\times$ congruency $X$ $\operatorname{SOA}[F(3,15)=11.97, p<.0001]$ and the nonsignificant interaction eccentricity $\times$ congruency $\times \operatorname{SOA}[F(3,15)=$ $1.86, p=.180]$. The corresponding interactions for error rate were not significant [size $\times$ congruency $\times$ SOA, $F(3,15)=1.56, p=.241$; eccentricity $\times$ congruency $\times$ SOA, $\left.F(3,15)=0.54, p_{\mathrm{HF}}=.663\right]$.

Clearly, the response inhibition effect jointly depended on prime size and eccentricity. Effects were strongest for large central stimuli, weakest for small peripheral stimuli, and intermediate for the remaining size-eccentricity combinations (Figure 5C). This is reflected in the reli-

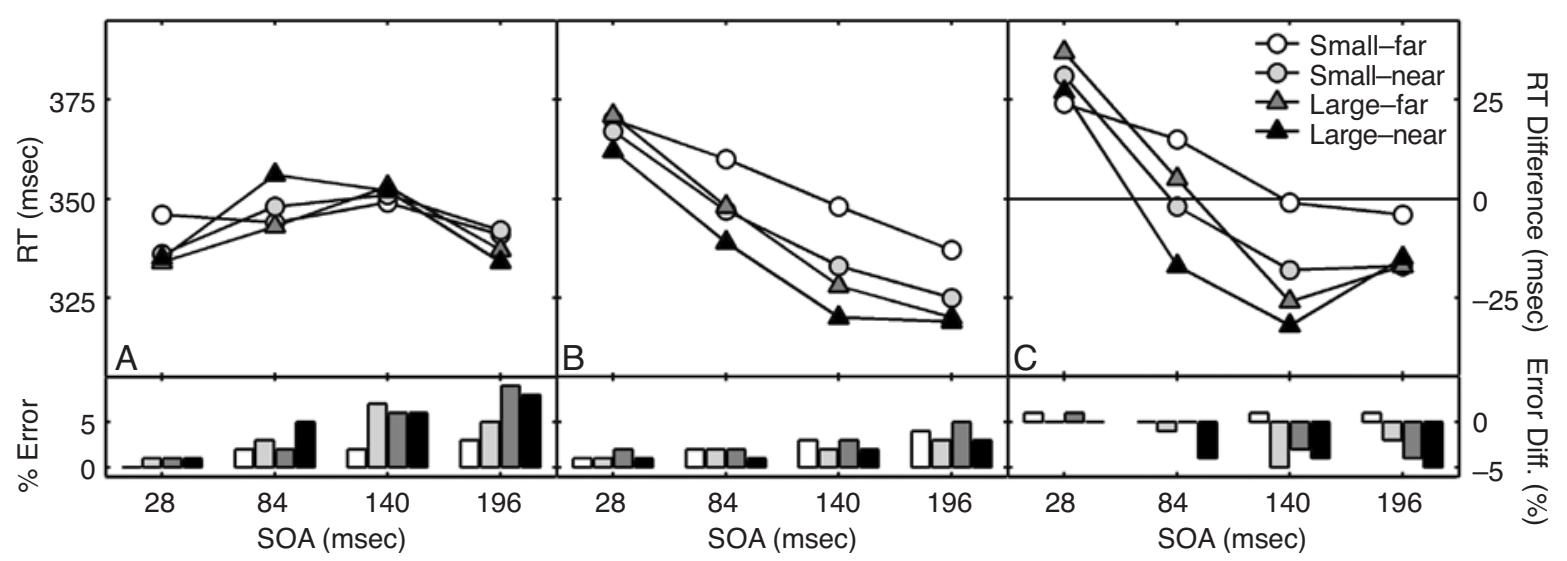

Figure 5. Response priming as a function of SOA, stimulus size, and eccentricity in Experiment 2. (A) Congruent trials. (B) Incongruent trials. (C) Net priming effect and error rate differences. For each column, the top panel shows mean response times (RTs) and the bottom panel shows error rates. 
able four-factor interaction size $\times$ eccentricity $\times$ congruency $\times$ SOA for $\mathrm{RT}[F(3,15)=4.19, p=.024]$. This interaction was not significant for error rate $[F(3,15)=$ $\left.4.48, p_{\mathrm{HF}}=.073\right]$. This ordering of effects is to be expected if the cortical magnification hypothesis holdsthat is, if response inhibition depends on prime size and eccentricity only via the extent of its cortical representation. Moreover, assuming that the cortical sizes of the stimuli were approximately matched at either eccentricity, the response inhibition time course should be the same for small central as for large peripheral primes. Figure 5 shows that both RTs and error rates indeed follow quite similar time courses, both on congruent and incongruent trials. These impressions are supported by the following additional statistical analyses.

Restricting the $2 \times 2$ size-eccentricity combinations to the small-near and large-far conditions eliminated all effects of eccentricity [eccentricity, $F(1,5)=4.30, p=$ .093 ; eccentricity $\times$ congruency, $F(1,5)=0.80, p=$ .414 ; eccentricity $\times \mathrm{SOA}, F(1,5)=1.14, p=.364$; eccentricity $\times$ congruency $\times$ SOA, $F(3,15)=1.15, p=$ .333]. These results are not just a consequence of reduced statistical power, as is evidenced by the fact that the response inhibition effect was still statistically reliable in the reduced ANOVA [congruency $\times$ SOA, $F(3,15)=$ 22.99, $p<.0005]$.

Likewise, computing the relevant contrasts (Howell, 2002) within the original ANOVA gave essentially the same results [eccentricity on congruent trials, $t(15)=0.497$, $p=.627$; eccentricity on incongruent trials, $t(15)=$ $0.186, p=.855$; eccentricity $\times$ congruency, $t(15)=$ $-0.843, p=.412$; eccentricity $\times$ congruency $\times$ SOA, $t(45)=0.258, p=.797]$. It seems safe to conclude that the response inhibition effects for large peripheral and small central primes follow the same time course.

As before, we checked whether these findings also hold at the level of the individual participant. Figure 6 shows the corresponding data, with cubic-spline interpolations added for clarity. The data are not as clear-cut as in Experiment 1, which may be because of the smaller SOA range and the different SOA spacing. Nevertheless, there was remarkable qualitative similarity between participants, in spite of quantitative shifts of the time course peaks and troughs. This is most noteworthy in the effects on incongruent trials, which show RT decreases with increasing SOA for all participants and all size-eccentricity conditions. In contrast, response slowing on congruent trials seems to arise with peaks at idiosyncratic SOA locations. As a consequence, little modulation is discernible in the average time course for small congruent primes at the far location (cf. Figure 5A), although post hoc trend tests revealed a significant cubic modulation with $\mathrm{SOA}[F(1,62)=8.72, p<$ $.01]$. In fact, all but one participant showed RT increases at some SOAs on congruent trials: Eight out of 18 possible comparisons revealed response slowing, in contrast to 0 out of 18 for incongruent trials $\left[\chi_{1}^{2}=7.88, p<.01\right]$. We can thus safely infer that even small congruent primes presented at the far eccentricity elicited response inhibition.

\section{Discussion}

Experiment 2 revealed reliable response inhibition effects at near and far eccentricities. First of all, in a replication of Experiment 1, there was evidence for response inhibition elicited by primes at a vertical eccentricity far beyond the value of $2.2^{\circ}$ deemed critical by Schlaghecken and Eimer (2000). The crucial new findings can be summarized as follows:

1. The time course of response inhibition is modulated both by prime eccentricity and prime size. As predicted, there is a large-small asymmetry in response inhibition that mirrors the central-peripheral asymmetry.

2. No reliable central-peripheral asymmetry remains if peripheral and central primes are matched with respect to cortical size.

These findings hold for the complete time courses, and not just at some particular SOA. Taken together, they provide strong evidence for the assumption that the size of a cortical representation determines the temporal dynamics of response inhibition. Thus, although response inhibition clearly depends on the specific combination of eccentricity and stimulus size, our data suggest that this dependence arises because the variables jointly determine the extent of the cortical representation. This is illustrated in Figure 7, which compares eccentricity effects for large and small stimuli (left) with size effects for stimuli at near and far locations (right). Obviously, reducing or increasing the size of the presumed cortical stimulus representation leads to almost identical effects on the response inhibition time course, whether the change in cortical size is achieved by changing stimulus eccentricity or stimulus size.

\section{GENERAL DISCUSSION}

The results of our experiments can be summarized as follows:

1. Peripheral and central visual stimuli produce effects of response inhibition on RT, with time courses modulated by both eccentricity and stimulus size.

2. Analysis of the individual time course data reveals that the timescale of inhibition changes with the strength of the cortical representation of the prime stimulus.

3 . No central-peripheral asymmetry of the effect remains when cortical magnification is controlled for.

Let us briefly comment on the implications of these findings. Reducing the size of the prime stimulus while keeping eccentricity fixed produced shifts in the time course analogous to those from presenting the stimulus more peripherally while keeping size constant. Such a tradeoff between the effects of eccentricity and size is suggestive of continuous mechanisms; it does not suggest the type of inhibition threshold proposed by Schlaghecken and Eimer (2002), which would imply a flat time course for primes at larger eccentricities unless substantial threshold variability is assumed. However, the time courses we observed in Experiment 1 were not only reduced in amplitude but also expanded toward larger mask-target de- 


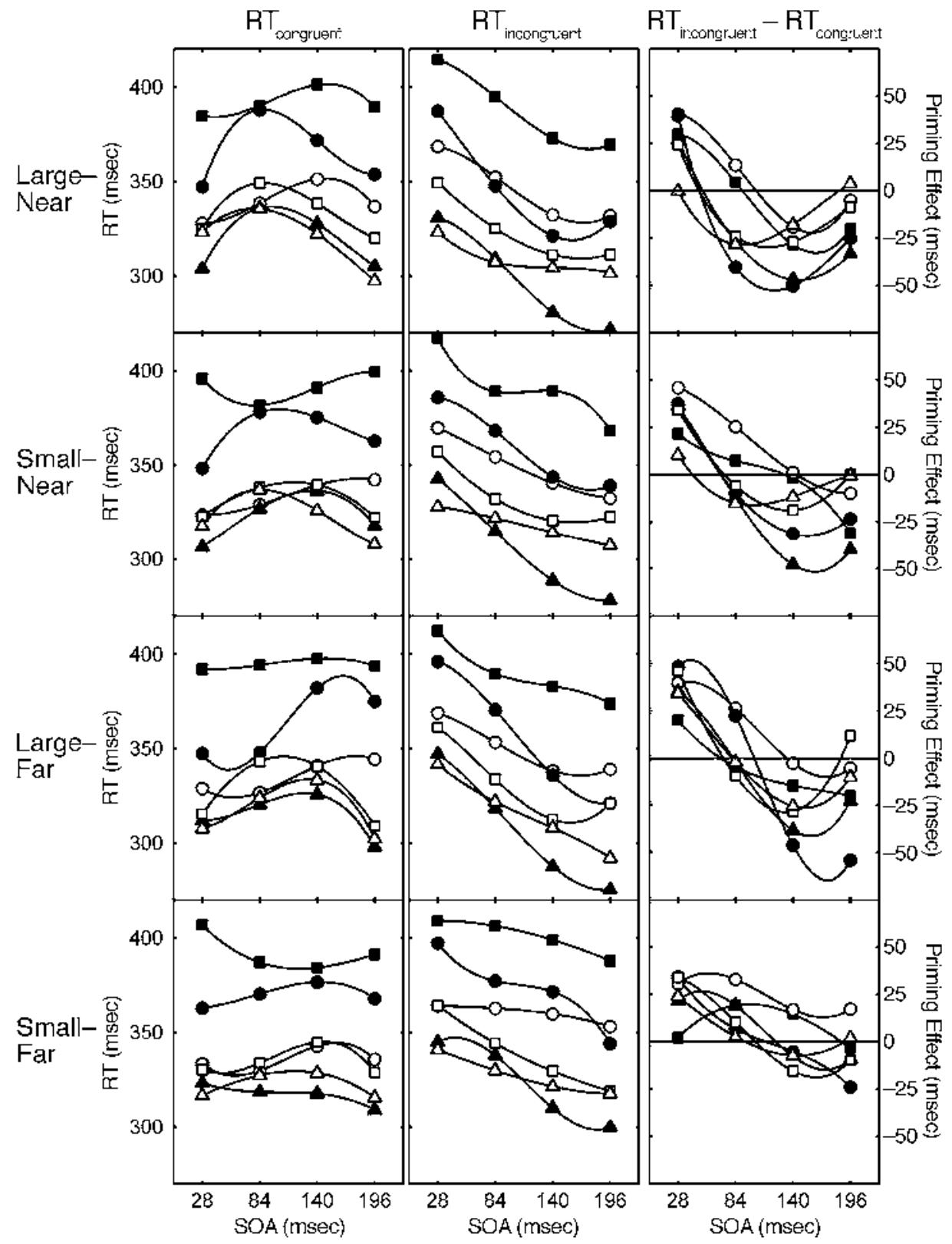

Figure 6. Time courses of individual participants in Experiment 2, with cubic splines fitted to the data. The left and middle columns show mean response times (RTs) on congruent and incongruent trials, respectively. The right column shows the net priming effect $\left(R T_{\text {incongruent }}-R_{\text {congruent }}\right)$. Rows indicate size $\times$ eccentricity combinations of primes.

lays as the prime appeared more peripherally. This change in the inhibition time course with stimulus eccentricity presents a challenge for threshold models.

Comparing the time courses for small-near and largefar primes provided support for the cortical magnification account, which assumes that the central-peripheral asymmetry reported by Schlaghecken and Eimer (1997) is merely a consequence of the fact that peripheral stimuli have smaller cortical representations than do central ones. If so, the activation and inhibition dynamics of stimuli across the visual field depend on eccentricity and physical size via cortical size only. In strong support of this view, almost identical time courses resulted when stimuli were adjusted for cortical magnification. This explains the empirical asymmetries without a threshold assumption.

We should note that although our results do not suggest the existence of a threshold, they also do not provide strong evidence against it. However, we do not believe that further studies of the central-peripheral asymmetry 


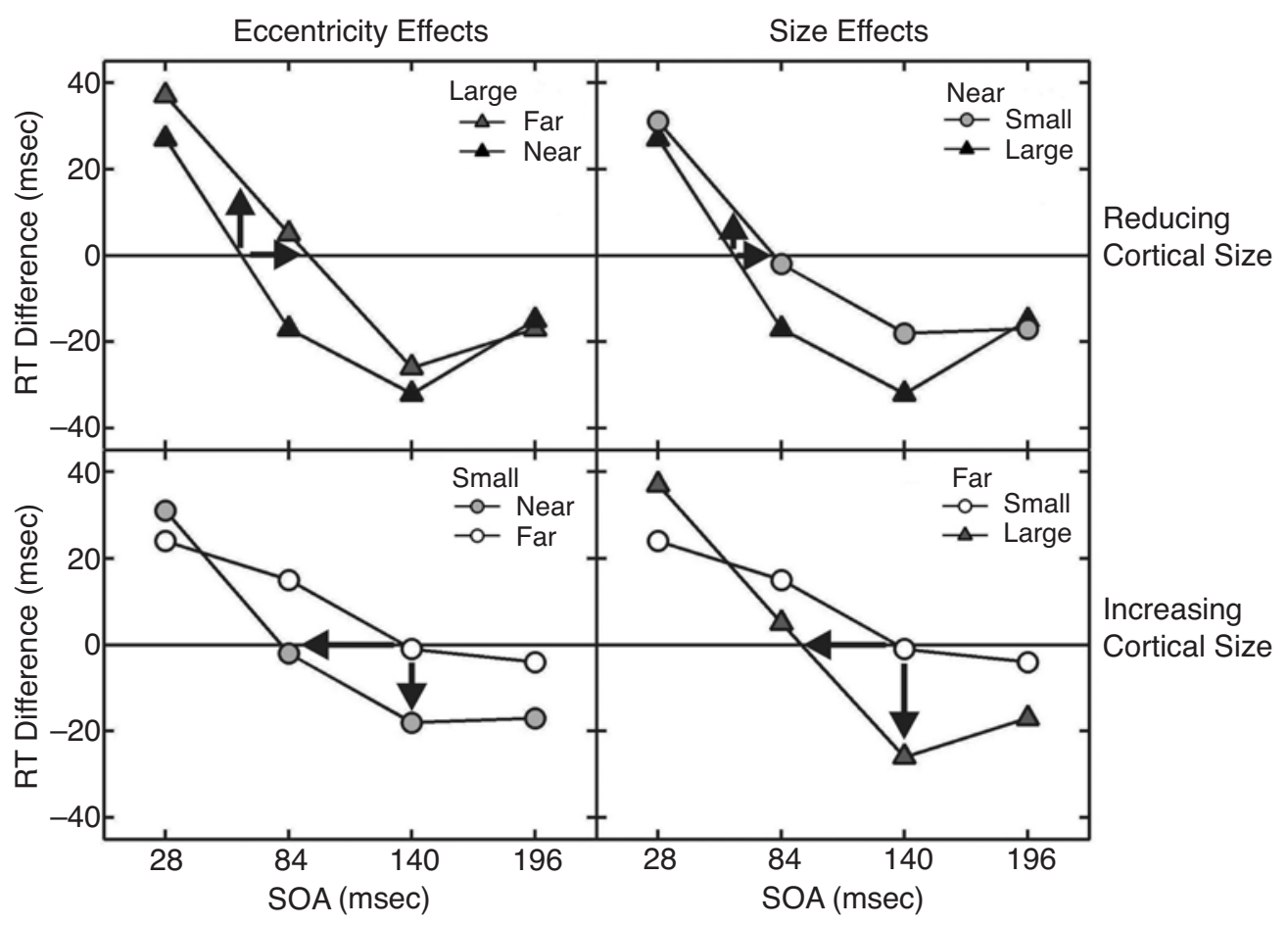

Figure 7. Effects of varying stimulus eccentricity (left) and size (right) on response inhibition time course. Arrows indicate that the effects of reducing (top panels) or increasing (bottom panels) presumed cortical size are analogous when size and eccentricity are manipulated. Eccentricity effects are for large (top) and small (bottom) stimuli. Size effects are for stimuli at near (top) and far (bottom) locations.

are likely to reveal specific information on the underlying inhibitory mechanisms, given that the asymmetry is but one example of many that follow from cortical magnification within the visual system (Strasburger, Rentschler, \& Harvey, 1994; but see Carrasco, McElree, Denisova, \& Giordano, 2003).

In fact, on the basis of the present results, not much can be inferred about where and how response inhibition arises. Therefore, we conclude this discussion with speculations on possible mechanisms underlying response inhibition, with remarks on research directions that seem promising for elucidating the response inhibition phenomenon in masked priming.

\section{Possible Mechanisms Underlying Inhibition}

As pointed out by Eimer and Schlaghecken (1998), response inhibition is likely to occur whenever perceptual information that initially gave rise to the activation of a particular motor response is no longer available. The question is when such a loss of perceptual information is detected. Recent studies stress the importance of feedback connections from extrastriate areas such as inferotemporal cortex and parietal cortex to early visual areas (Lamme \& Roelfsema, 2000; Van Essen \& DeYoe, 1995). According to Lamme and Roelfsema, after the appearance of a visual stimulus, several feed-forward processing sweeps start operating in parallel but at different speeds. A quick-and-dirty sweep performs the coarse analysis of visual features, and a slow sweep operates at much higher resolution. As a result, higher visual areas will already have received preliminary data about the stimulus when the next, more detailed sweep arrives. Both Lamme and Roelfsema (2000) and Di Lollo, Enns, and Rensink (2000) propose that activitation at higher visual areas is matched against that at earlier visual areas in order to relate it to the corresponding locations in visual space. Therefore, the detection of a mismatch in the information at earlier and later cortical sites might underlie the phenomenon of visual masking.

Let us assume that whenever such a mismatch is detected, any task-related neural activity that was elicited by a masked (and nonperceived) stimulus must be inhibited. This could be achieved either by a general reset signal or, more specifically, by inhibiting the respective response units in inverse proportion to their current activation. The latter possibility seems more economical and could easily account for the observed response slowing after congruent primes and the speed-up after incongruent primes if the response units are assumed to inhibit each other reciprocally. Spreading of inhibition takes time, as can be seen from the performance benefits on congruent trials observed at 0 SOA (i.e., when mask and target are identical). Only after about $50 \mathrm{msec}$ does the benefit from congruent primes begin to turn into a disadvantage and the RT cost from incongruent primes turn into an advantage. How strongly a motor response is activated by 
the time a mismatch is detected will critically depend on the representational strength of the prime stimulus, with stronger representations leading to larger activations. Therefore, weakly represented stimuli will lead to smaller performance benefits at any given SOA and will require more time for response inhibition to build up than will stimuli with stronger representations.

These assumptions are speculative and require further investigation. Note, however, that the predictions that follow from them are in agreement both with the RT and error rate time courses found here and the LRP time course data reported by Eimer and Schlaghecken (1998). Nevertheless, we think that a more thorough understanding of the mechanisms underlying response inhibition requires considering the following issues:

1. According to the theories of Lamme and Roelfsema (2000) and Di Lollo, Enns, and Rensink (2000), response inhibition critically depends on both prime-mask and mask-target SOAs. Whereas the delay between prime and mask determines the amount of motor activation by the time the mask arrives, the delay between mask and target determines the time available for inhibition to develop. Therefore, studying the joint effects of these two temporal variables seems indispensable for understanding the basic mechanisms of response inhibition. As was shown in our experiments, even if the strength of the inhibitory signal is insufficient for producing an NCE, contrasting the time courses for congruent and incongruent trials may reveal traces of response inhibition that are missed in experiments with SOA fixed. Obviously, examining time course data helps avoid the fallacy of taking the absence of an NCE for proof of the lack of response inhibition. Moreover, parametric studies of the temporal stimulus factors are likely to provide data that are crucial for deciding between alternative quantitative models of inhibition.

2 . If response inhibition is triggered by a mismatch detection mechanism, the mask should play a substantial role. At present, there seems to be consensus that response inhibition occurs only if the prime is made invisible by the mask (Klapp \& Hinkley, 2002). However, in contrast to the effects that have been found for primes followed by either a strong or an inefficient metacontrast mask, we have found clear evidence for response inhibition elicited by primes that are fully visible (Vorberg, 1998, 2000). Two factors seem to contribute to this apparent disagreement: First, the typical approach to manipulating prime visibility is to replace the mask with a blank interval (Klapp \& Hinkley, 2002). Such a comparison is problematic, however, if the onset of the mask is crucial for the occurrence of response inhibition. In our previous experiments, we therefore used a pseudomaskthat is, a nonmasking stimulus that does not obliterate the prime stimulus (e.g., an open rectangle). Second, when assessing the full time courses, we observed that fully visible primes did elicit NCEs, but with onset delayed in comparison with the NCE from invisible primes. If we had kept SOA constant (Klapp \& Hinkley, 2002), we would have come to the conclusion that no response in- hibition derived from consciously perceived stimuli. This difference in results again demonstrates the importance of considering full time course information rather than focusing on an arbitrarily selected time point.

3. A current debate concerns whether the observed NCE does in fact reflect active inhibition of the response facilitated by the prime, or rather an activation of the opposite response. The latter possibility has some plausibility, because the pattern mask introduced by Eimer and Schlaghecken (1998) is formed by the superimposition of the two prime stimuli, and altering the shape of the mask may reduce or fully eliminate the NCE (Lleras \& Enns, 2004; Verleger, Jaskowski, Aydemir, van der Lubbe, $\&$ Groen, 2004). The argument that the NCE is tied to the particular type of pattern mask seems weakened by the present findings that an NCE can also be demonstrated under metacontrast masking conditions. We hasten to add, however, that the above objection might also apply to our stimuli, as the central cutout in the mask stimulus is formed by the contours of the superimposed opposite arrow primes (see Figure 2). It is thus conceivable that presentation of the mask indirectly activates the perceptual representation opposite to that of the prime stimulus (e.g., via the holes left open when prime and mask are superimposed). This activation would facilitate target processing on incongruent trials (because it is congruent with the opposite representation) but interfere with processing on congruent trials. Although we do not consider this possibility to be likely, it cannot be ruled out at present. However, some evidence does favor response inhibition rather than opposite-response activation: Using three rather than two response alternatives, Klapp and Hinkley (2002) nevertheless observed evidence for response inhibition. Similarly, Eimer and Schlaghecken (1998) found response inhibition in a go/no-go task. On the basis of these results, the NCE is unlikely to be due to excitation of the opposite response unit only. Our methodological objection is by now well known: The evidence in favor of opposite-response activation is mostly from experiments that contrasted the effects of different types of masks at a fixed mask-target SOA, without taking into consideration the possibility of shifts in the respective time courses. Again, clarification of the issue will require experiments that vary the temporal aspects of the stimuli. We therefore conclude with some methodological recommendations.

Experiments that vary the durations of prime, mask, and/or target stimuli and the onset asynchronies between them require careful consideration of the following issues:

With SOA constant within blocks, SOA effects may reflect strategic adaptations to the particular conditions rather than effects of the temporal variable; thus, time course data may be contaminated with strategic effects. There is ample evidence in the literature on RT foreperiod effects (Luce, 1986) that such contamination is likely to occur. Even more relevant is the recent demonstration by Naccache, Blandin, and Dehaene (2002) that the magnitude of subliminal priming is modulated on the basis of whether the prime stimulus falls into a predictable time 
window or not. However, full randomization may be problematic if large SOA ranges are studied, because participants may adjust strategies to the longer waits, which would distort short-range effects. A good compromise would be to randomize SOA within limitedrange subsets.

Also, the temporal dynamics of priming and inhibition effects may vary substantially between participants. An example can be seen in Figure 4 (right panel), which shows that at $\mathrm{SOA}=168 \mathrm{msec}$, far congruent primes led to RT costs for half of the participants but RT benefits for the other half. Averaging across participants is bound to give misleading results unless individual time courses differ in amplitude only, an assumption that is clearly unwarranted. Thus, analyzing time courses separately for participants is desirable.

We believe that in spite of their additional demands, parametric studies of the time course of masked priming are crucial for revealing the cognitive architecture that underlies information processing with and without awareness.

\section{REFERENCES}

Carrasco, M., \& Frieder, K. S. (1997). Cortical magnification neutralizes the eccentricity effect in visual search. Vision Research, 37, 63-82.

Carrasco, M., McElree, B., Denisova, K., \& Giordano, A. M. (2003). Speed of visual processing increases with eccentricity. Nature Neuroscience, 6, 669-670.

DAYAn, P., \& Aввотт, L. F. (2001). Theoretical neuroscience: Computational and mathematical modeling of nervous systems. Cambridge, MA: MIT Press.

DeValois, R. L., \& DeValois, K. K. (1988). Spatial vision. New York: Oxford University.

Di Lollo, V., Enns, J. T., \& Rensink, R. A. (2000). Competition for consciousness among visual events: The psychophysics of reentrant visual processes. Journal of Experimental Psychology: General, 129, 481-507.

Eimer, M., \& Schlaghecken, F. (1998). Effects of masked stimuli on motor activation: Behavioral and electrophysiological evidence. Journal of Experimental Psychology: Human Perception \& Performance, 24, 1737-1747.

Howell, D. C. (2002). Statistical methods for psychology (5th ed.). Pacific Grove, CA: Duxbury.

KlaPP, S. T., \& HinkLEy, L. B. (2002). The negative compatibility effect: Unconscious inhibition influences reaction time and response selection. Journal of Experimental Psychology: General, 131, 255-269.

Klotz, W., \& WolfF, P. (1995). The effect of a masked stimulus on the response to the masking stimulus. Psychological Research, 58, 92-101.

LAmme, V. A., \& Roelfsema, P. R. (2000). The distinct modes of vision offered by feedforward and recurrent processing. Trends in Neurosciences, 23, 571-579.

Lleras, A., \& ENNS, J. T. (2004). Negative compatibility or object updating? A cautionary tale of mask-dependent priming. Journal of Experimental Psychology: General, 133, 475-493.

LuCE, R. D. (1986). Response times. New York: Oxford University Press.
Naccache, L., Blandin, E., \& Dehaene, S. (2002). Unconscious masked priming depends on temporal attention. Psychological Science, 13, 416-424.

Neumann, O., \& Klotz, W. (1994). Motor responses to non-reportable, masked stimuli: Where is the limit of direct parameter specification? In C. Umiltà \& M. Moscovitch (Eds.), Attention and performance $X V$ : Conscious and nonconscious information processing (pp. 123150). Cambridge, MA: MIT Press, Bradford Books.

Schlaghecken, F., \& Eimer, M. (1997). The influence of subliminally presented primes on response preparation. Sprache \& Kognition, 16, $166-175$.

Schlaghecken, F., \& EIMER, M. (2000). A central-peripheral asymmetry in masked priming. Perception \& Psychophysics, 62, 1367-1382.

Schlaghecken, F., \& Eimer, M. (2002). Motor activation with and without inhibition: Evidence for a threshold mechanism in motor control. Perception \& Psychophysics, 64, 148-162.

Sereno, M. I., Dale, A. M., Reppas, J. B., Kwong, K. K., Belliveau, J. W., Brady, T. J., Rosen, B. R., \& Tootell, R. B. H. (1995). Borders of multiple visual areas in humans revealed by functional magnetic resonance imaging. Science, 268, 889-893.

Slotnick, S. D., Klein, S. A., Carney, T., \& Sutter, E. E. (2001). Electrophysiological estimate of human cortical magnification. Clinical Neurophysiology, 112, 1349-1356.

Strasburger, H., Rentschler, I., \& Harvey, L. O. (1994). Cortical magnification theory fails to predict visual recognition. European Journal of Neuroscience, 6, 1583-1588.

VAN Essen, D. C., \& DeYoe, E. A. (1995). Concurrent processing in the primate visual cortex. In M. S. Gazzaniga (Ed.), The cognitive neurosciences (pp. 383-400). Cambridge, MA: MIT Press.

Verleger, R., Jaskowski, P., Aydemir, A., van der Lubbe, R. H. J., \& GROEN, M. (2004). Qualitative differences between conscious and nonconscious processing? On inverse priming induced by masked arrows. Journal of Experimental Psychology: General, 133, 494-515.

VORBERG, D. (1998). Reaktionen auf unbewusste visuelle Reize: Umkehr von Bahnung in Hemmung [Abstract]. In H. Lachnit, A. Jacobs, \& F. Rösler (Eds.), Experimentelle psychologie (p. 386). Marburg: Pabst. VORBERG, D. (2000). Wann wirken bewusste Reize anders als unbewusste? [Abstract]. In H. H. Bülthoff, M. Fahle, K. R. Gegenfurtner, \& H. A. Mallot (Eds.), Beitrage zur 3. Tübinger Wahrnehmungskonferenz (p. 33). Tübingen: Knirsch.

Vorberg, D., Mattler, U., Heinecke, A., Schmidt, T., \& SchwarzBACH, J. (2003). Different time courses for visual perception and action priming. Proceedings of the National Academy of Sciences, 100, 6275-6280.

Vorberg, D., Mattler, U., Heinecke, A., Schmidt, T., \& SchwarzBACH, J. (2004). Invariant time course of priming with and without awareness. In C. Kaernbach, E. Schröger, \& H. Müller (Eds.), Psychophysics beyond sensation: Laws and invariants of human cognition. Mahwah, NJ: Erlbaum.

WILCOX, R. (1997). Introduction to robust estimation and hypothesis testing. San Diego: Academic Press.

\section{NOTE}

1. There is little agreement on the terminology to use for the relation between target and prime stimuli. Across - but not within - experimental paradigms, congruency, congruity, compatibility, consistency, and correspondence can be seen almost as synonyms. We conform with the usage established within the masked priming literature and use congruency, congruent, and incongruent to refer to the prime-target relationship concerning the response mapped to the relevant stimulus feature. 


\section{APPENDIX}

Empirically, the cortical magnification $M$ at eccentricity $E$ has been shown to be approximated well by the function $M(E)=\Delta x / \Delta E=A /\left(E+E_{2}\right)$ (Slotnick, Klein, Carney, \& Sutter, 2001), where $\Delta x$ (in millimeters) is the change in cortical size of a stimulus, $\Delta E$ (in degrees) is the change in its eccentricity, and $E_{2}$ (in degrees) is the eccentricity at which the corresponding representation in visual cortex is half as large as for central presentation; $A$ (in millimeters) is a constant cortical scaling factor. Estimates for $E_{2}$ and $A$ vary greatly between studies, depending on the measurement method. Slotnick et al. have summarized recent studies, both from animal and human research. We averaged the estimates they reported for human participants studied either with fMRI (Sereno et al., 1995) or with dipole source localization (Slotnick et al., 2001), discarding estimates that were obvious outliers. This resulted in values of $E_{2}=0.404$ and $A=19.43$ as estimates.

Applying the inverse cortical magnification factor $M^{-1}$ to the stimuli of Experiment 2 gives a required ratio of the stimulus sizes at the near and far eccentricities of 1 to 2.57 . Because of constraints imposed by screen resolution and stimulus geometry, this exact ratio was not feasible. We opted for 1:2 as the nearest size ratio that could be achieved for the vertical extent of the prime stimuli, and 1:1.4 for that of the targets, which was of lesser importance.

(Manuscript received February 17, 2004;

revision accepted for publication August 11, 2004.) 